\title{
Light bullets and optical collapse in vacuum
}

\author{
Gert Brodin ${ }^{a}$, Lennart Stenflo ${ }^{a}$, Dan Anderson ${ }^{\mathrm{b}}$, Mietek Lisak ${ }^{\mathrm{b}}$, \\ Mattias Marklund ${ }^{\mathrm{b}}$ and Pontus Johannisson ${ }^{\mathrm{b}}$ \\ ${ }^{a}$ Department of Plasma Physics, Umeå University, SE-901 87 Umeå, Sweden \\ ${ }^{\mathrm{b}}$ Department of Electromagnetics, Chalmers University of Technology, SE-412 96 \\ Göteborg, Sweden
}

\begin{abstract}
In quantum electrodynamics, photon-photon scattering can be the result of the exchange of virtual electron-positron pairs. Effectively, this gives rise to self-interaction terms in Maxwell's equations, similar to the nonlinearities due to polarization in nonlinear optics. These self-interaction terms vanish in the limit of parallel propagating waves. However if the modes generated in bounded regions are used, there will be a non-zero total effect. We show that stationary two-dimensional light bullets can form in guiding structures, due to the balancing effect of quantum electrodynamical vacuum nonlinearities on dispersion and diffraction. These light bullets are unstable and exhibit the possibility of self-focusing collapse. The consequences of our results are also discussed.
\end{abstract}

PACS numbers: 12.20.Ds, 42.50.Vk, 42.65.T

\section{Introduction}

According to quantum electrodynamics (QED), the non-classical phenomenon of photon-photon scattering can take place due to the exchange of virtual electron-positron pairs. This is a second order effect (in terms of the fine structure constant $\left.\alpha \equiv e^{2} / 4 \pi \varepsilon_{0} \hbar c \approx 1 / 137\right)$, which in standard notation can be formulated in terms of the Euler-Heisenberg Lagrangian density $[1,2]$

$$
\mathscr{L}_{\mathrm{EH}}=\varepsilon_{0} \mathscr{F}+\xi\left(4 \mathscr{F}^{2}+7 \mathscr{G}^{2}\right),
$$

where $\xi \equiv 2 \alpha^{2} \varepsilon_{0}^{2} \hbar^{3} / 45 m_{e}^{4} c^{5}, \mathscr{F} \equiv \frac{1}{2}\left(E^{2}-c^{2} B^{2}\right), \mathscr{G} \equiv c \mathbf{E} \cdot \mathbf{B}, e$ is the electron charge, $c$ the velocity of light, $2 \pi \hbar$ the Planck constant and $m_{e}$ the electron mass. The latter terms in expression (1) represent the effects of vacuum polarization and magnetization. We note that $\mathscr{F}=\mathscr{G}=0$ in the limit of parallel propagating waves. It is therefore necessary to use other waves in order to 
obtain an effect from the QED corrections. Furthermore, it has recently been shown that QED vacuum nonlinearities can lead to self-focusing of laser beams [3], and that such effects might be experimentally viable within a decade. In Ref. [4] it was found that guiding structures can be useful for the purpose of detecting QED effects, and a suggestion was presented for an experimental setup that gives a measurable signal.

In the present work we thus consider waves guided by two parallel conducting planes, in order to study the possibility of using nonlinear QED effects to balance diffraction and dispersion in directions parallel to the planes. We find that our system is governed by a $1+2$ dimensional nonlinear Schrödinger equation (NLSE). Using approximate variational methods, which have proven to be useful in similar situations (see e.g. $[5,6]$ ), we find that the nonlinearities can counteract diffraction and dispersion to form 2-dimensional light bullet solutions, i.e., solitary solutions to the NLSE which preserve the envelopes of the fields. The formation of light bullets was in other contexts (optical media) first studied by Silberberg [7].

\section{Light bullet solutions}

In a medium with polarization $\mathbf{P}$ and magnetization $\mathbf{M}$, the general wave equations for $\mathbf{E}$ and $\mathbf{B}$ are [4]

$$
\frac{1}{c^{2}} \frac{\partial^{2} \mathbf{E}}{\partial t^{2}}-\nabla^{2} \mathbf{E}=-\mu_{0}\left[\frac{\partial^{2} \mathbf{P}}{\partial t^{2}}+c^{2} \nabla(\nabla \cdot \mathbf{P})+\frac{\partial}{\partial t}(\nabla \times \mathbf{M})\right]
$$

and

$$
\frac{1}{c^{2}} \frac{\partial^{2} \mathbf{B}}{\partial t^{2}}-\nabla^{2} \mathbf{B}=\mu_{0}\left[\nabla \times(\nabla \times \mathbf{M})+\frac{\partial}{\partial t}(\nabla \times \mathbf{P})\right] .
$$

Furthermore, the effective polarization and magnetization in vacuum due to photon-photon scattering induced by the exchange of virtual electron-positron pairs are given by (see, e.g., Ref. [3])

$$
\mathbf{P}=2 \xi\left[2\left(E^{2}-c^{2} B^{2}\right) \mathbf{E}+7 c^{2}(\mathbf{E} \cdot \mathbf{B}) \mathbf{B}\right]
$$

and

$$
\mathbf{M}=-2 c^{2} \xi\left[2\left(E^{2}-c^{2} B^{2}\right) \mathbf{B}+7(\mathbf{E} \cdot \mathbf{B}) \mathbf{E}\right] .
$$

We consider propagation between two parallel conducting planes with spacing $x_{0}$ (i.e., the region $0 \leq x \leq x_{0}$ is vacuum surrounded by the plates that, as a starting point, are assumed to be perfectly conducting). We assume that only one $\mathrm{TE}_{n 0}$ mode $(n=1,2, \ldots)$ is present. To linear order, this gives the fields 


$$
\begin{aligned}
& B_{z}=\frac{\pi}{x_{0}} \tilde{A} \cos \left(\frac{n \pi x}{x_{0}}\right) \exp [\mathrm{i}(k z-\omega t)]+\text { c.c., } \\
& B_{x}=-\mathrm{i} k \tilde{A} \sin \left(\frac{n \pi x}{x_{0}}\right) \exp [\mathrm{i}(k z-\omega t)]+\text { c.c. } \\
& E_{y}=\mathrm{i} \omega \tilde{A} \sin \left(\frac{n \pi x}{x_{0}}\right) \exp [\mathrm{i}(k z-\omega t)]+\text { c.c. }
\end{aligned}
$$

together with $0 \approx \omega^{2} / c^{2}-k^{2}-n^{2} \pi^{2} / x_{0}^{2}$. Here c.c. stands for complex conjugate.

It should be noted that the beams propagate with a 90 degree angle with respect to each other for the selfconsistent solutions obtained in a similar situation in Ref. [3]. This would correspond to the relation $k=n \pi / x_{0}$ in our case. However, we have no such limitation in our derivation. The result of a 90 degree beam angle of Ref. [3] comes from requiring an explicit symmetry between the wave equations for $\mathbf{E}$ and $\mathbf{B}$. A similar requirement is not needed in our case, since the $\mathrm{TE}_{n 0}$-modes introduced by the conducting planes do not have a complete electric-magnetic symmetry as, for example, the electric fields vanish close to the planes. What one still might worry about is whether or not both Faraday's and Ampere's law, which are equivalent to the two wave equations for $\mathbf{E}$ and $\mathbf{B}$, are properly solved in our case. However, the equation that will be derived for the vector potential is equivalent to Ampere's law, which is equivalent to the wave equation for the electric field, and using the vector potential we therefore automatically solve Faraday's law. It thus follows that our solution for the vector potential corresponds to electromagnetic fields that are consistent with the two wave equations for $\mathbf{E}$ and $\mathbf{B}$.

We have expressed the fields in terms of the vector potential amplitude $\tilde{A}$, given by $\mathbf{A}=(0, A, 0)$, where

$$
A=\tilde{A} \sin \left(\frac{n \pi x}{x_{0}}\right) \exp [\mathrm{i}(k z-\omega t)]+\text { c.c. }
$$

using the radiation gauge $(\phi=0)$. A nonlinear dispersion relation can be derived by inserting the linear expression for the fields into the right hand side of Eq. (3), taking the $z$-component of the resulting equation, and separating into orthogonal trigonometric functions. From that equation the coefficients in the NLSE can be found. However, here we will present an alternative derivation, starting directly from the Euler-Heisenberg Lagrangian (1), which is more elegant and gives the same result. Naturally we must first express $\mathscr{L}_{\mathrm{EH}}$ in terms of the electromagnetic potential, since it is the basis for the original variational principle. We take $\tilde{A}=\tilde{A}(t, z)$, and assume $\tilde{A}$ to be weakly modulated $(|\partial \tilde{A} / \partial t| \ll|\omega \tilde{A}|,|\partial \tilde{A} / \partial z| \ll|k \tilde{A}|)$, but we omit the slow $y$-dependence, since such a dependence makes the derivation technically more complicated without adding extra understanding. A diffraction term in the $y$-direction is lost by this procedure, but this effect is trivially added afterwards. To lowest order, we thus omit the nonlinear terms and the slow derivatives in $\mathscr{L}$. Noting that 
$\mathscr{F}=(1 / 2)\left[(\partial A / \partial t)^{2}-c^{2}(\nabla A)^{2}\right]$ and $\mathscr{G}=0$, we find that the non-oscillating part (i.e. the part that does not vanish after integration) is

$$
\mathscr{L}_{0} / \varepsilon_{0} \equiv \mathscr{F}=\frac{1}{2}\left(\omega^{2}-k^{2} c^{2}\right)|\tilde{A}|^{2} \sin ^{2}\left(\frac{n \pi x}{x_{0}}\right)-\frac{n^{2} \pi^{2} c^{2}}{2 x_{0}^{2}}|\tilde{A}|^{2} \cos ^{2}\left(\frac{n \pi x}{x_{0}}\right) .
$$

After performing the integration over the region between the plates we find that the lowest order part of the action vanishes identically as the dispersion relation is here considered to be satisfied. Going to the next order of approximation in the Lagrangian we include first order slow derivatives, which yields $\mathscr{L}_{1} / \varepsilon_{0}=\mathscr{L}_{0} / \varepsilon_{0}+\mathrm{i} \omega\left[(\partial A / \partial t) A^{*}-\left(\partial A^{*} / \partial t\right) A\right]-\mathrm{i} k c^{2}\left[(\partial A / \partial z) A^{*}-\left(\partial A^{*} / \partial z\right) A\right]$. After variation this leads to an equation where the envelope moves with the group velocity. The next order and final approximation includes second order slow derivatives and the $\mathscr{F}^{2}$ term in the Lagrangian. After performing the $x$-integration, dropping $\mathscr{L}_{0}$ (since the action is identically zero because of the dispersion relation) and eliminating the second order slow time-derivatives using $\partial^{2} / \partial t^{2} \approx v_{g}^{2} \partial^{2} / \partial z^{2}$, the final expression for the Lagrangian is

$$
\begin{array}{r}
\left\langle\mathscr{L}_{2}\right\rangle=\mathrm{i} \omega \varepsilon_{0}\left(\frac{\partial \tilde{A}}{\partial t} \tilde{A}^{*}-\frac{\partial \tilde{A}^{*}}{\partial t} \tilde{A}\right)-\mathrm{i} k c^{2} \varepsilon_{0}\left(\frac{\partial \tilde{A}}{\partial z} \tilde{A}^{*}-\frac{\partial \tilde{A}^{*}}{\partial z} \tilde{A}\right) \\
+\left(c^{2}-v_{g}^{2}\right) \varepsilon_{0}\left|\frac{\partial \tilde{A}}{\partial z}\right|^{2}+\frac{3 n^{4} c^{4} \pi^{4} \xi}{x_{0}^{4}}|\tilde{A}|^{4},
\end{array}
$$

where \langle\rangle stands for integration over $x$. Variation with respect to $\tilde{A}^{*}$ leads to

$$
2 \mathrm{i} \omega\left(\frac{\partial}{\partial t}+v_{g} \frac{\partial}{\partial z}\right) A+c^{2} \frac{\partial^{2} A}{\partial y^{2}}+\omega v_{g}^{\prime} \frac{\partial^{2} A}{\partial z^{2}}+\frac{6 n^{4} c^{4} \pi^{4} \xi}{\varepsilon_{0} x_{0}^{4}}|A|^{2} A=0,
$$

where $v_{g}$ is the group velocity and $v_{g}^{\prime}$ the group dispersion that follows from the linear dispersion relation. We have also added the diffraction term in the $y$-direction corresponding to the full amplitude dependence $A=\tilde{A}(t, y, z)$. Changing to a system moving with the group velocity while rescaling the coordinates and the amplitude according to

$$
\begin{array}{rlrl}
\tau & =\omega t / 2, & v & =\omega y / c, \\
\xi & =\sqrt{\omega / v_{g}^{\prime}}\left(z-v_{g} t\right), a & =\sqrt{\frac{6 n^{4} c^{4} \pi^{4} \xi}{\omega \varepsilon_{0} x_{0}^{4}}} A,
\end{array}
$$

we obtain

$$
\mathrm{i} \frac{\partial a}{\partial \tau}+\frac{\partial^{2} a}{\partial v^{2}}+\frac{\partial^{2} a}{\partial \xi^{2}}+|a|^{2} a=0
$$

which corresponds to the rescaled Lagrangian density

$$
\mathscr{L}=\frac{\mathrm{i}}{2}\left(a^{*} \frac{\partial a}{\partial \tau}-a \frac{\partial a^{*}}{\partial \tau}\right)-\left|\frac{\partial a}{\partial v}\right|^{2}-\left|\frac{\partial a}{\partial \xi}\right|^{2}+\frac{1}{2}|a|^{4},
$$


where the diffraction term in the $y$-direction is also included.

From now on we will look for cylindrically symmetric solutions of Eq. (10), i.e., $a=a(t, \rho)$, where $\rho^{2}=v^{2}+\xi^{2}$. Equation (10) can then be written

$$
\mathrm{i} \frac{\partial a}{\partial \tau}+\frac{1}{\rho} \frac{\partial}{\partial \rho}\left(\rho \frac{\partial a}{\partial \rho}\right)+|a|^{2} a=0,
$$

while the Lagrangian density (11) takes the form

$$
\mathscr{L}=\frac{\mathrm{i}}{2}\left(a^{*} \frac{\partial a}{\partial \tau}-a \frac{\partial a^{*}}{\partial \tau}\right)-\left|\frac{\partial a}{\partial \rho}\right|^{2}+\frac{1}{2}|a|^{4},
$$

with the action given by $\mathscr{A}=\int \mathscr{L} \rho d \rho d \tau$. Equation (12) is a 2-dimensional radially symmetric NLSE. Although exact solutions of this equation are not available, numerical and approximate analysis give a clear picture of the solutions. In particular, it has been shown that Eq. (12) allows a stationary solution where the diffractive/dispersive spreading of the pulse in the coordinate $\rho$ is balanced by the focusing effect created by the nonlinearity. An accurate analytical approximation of the dynamics of the pulse-like solutions of Eq. (12) can be obtained using direct variational methods involving the Lagrangian $\mathscr{L}$ given by expression (13) and subsequent Rayleigh-Ritz optimization based on suitably chosen trial functions (see e.g. [6] and references therein). A convenient trial function for the present problem is [5]

$$
a_{T}(\tau, \rho)=F(\tau) \operatorname{sech}\left[\frac{\rho}{f(\tau)}\right] \exp \left[\mathrm{i} b(\tau) \rho^{2}\right]
$$

which involves a complex amplitude $F(\tau)$, a pulse width $f(\tau)$, and a quadratic phase function modeling the phase front curvature. Inserting this ansatz into the variational integral and integrating over $\rho$, a reduced variational problem is obtained for the parameter functions, $F(\tau), F^{*}(\tau), f(\tau)$ and $b(\tau)$. The subsequent reduced Euler-Lagrange equations can be rearranged to give $F$, $F^{*}$ and $b$ as explicit functions of the width $f(\tau)$, which satisfies the equation

$$
\frac{d^{2} f}{d \tau^{2}}=\gamma\left(1-\frac{I}{I_{c}}\right) \frac{1}{f^{3}}
$$

where $\gamma=4(\ln 4+1) /(27 \zeta(3)) \simeq 0.29, I(\tau)=f^{2}(\tau)|F(\tau)|^{2}=f^{2}(0)|F(0)|^{2}=$ $I_{0}$, and $I_{c}=(2 \ln 2+1) /(4 \ln 2-1) \simeq 1.35$. Obviously, stationary solutions exist when the pulse power satisfies $I_{0}=I_{c}$. Furthermore, the full solution of Eq. (15) is

$$
f(\tau)=f(0) \sqrt{1+\frac{\gamma}{f^{2}(0)}\left(1-\frac{I_{0}}{I_{c}}\right) \tau^{2}},
$$

$\overline{1 \text { Here }} \zeta(p)$ is the Riemann zeta function. 
which shows that the stationary solution is unstable and either collapses to zero width in a finite time when $I_{0}>I_{c}$, or diffracts monotonously towards infinite width when $I_{0}<I_{c}$.

\section{Discussion and conclusion}

From Sec. 2, it is clear that the most interesting alternative is $I_{0}>I_{c}$, in which case the QED vacuum nonlinearities play a crucial role, leading to a collapse. In dimensional units the inequality $I_{0}>I_{c}$ roughly leads to $E_{\text {init }}^{2} k^{2} r_{\text {init }}^{2}>E_{\text {crit }}^{2}$, where $E_{\text {init }}$ and $r_{\text {init }}$ are the initial electric field and beam radius, respectively, and the critical QED electric field is defined by $E_{\text {crit }}^{2}=\varepsilon_{0} / \xi$. Naturally this unbounded self-focusing will eventually be saturated by some kind of higher order nonlinear mechanism. However, this will not occur before an electric field level of order $E \sim E_{\text {crit }}$ is reached, which is the field strength when both our perturbative nonlinear calculation scheme and our starting expression, the Euler-Heisenberg Lagrangian, breaks down. For such extreme energy densities, higher order Feynman diagrams must be included in the QED description, and possibly the corresponding physical effects may then counteract the collapse scenario, resulting in a saturated beam radius $r_{\text {sat }} \sim r_{\text {init }} E_{\text {init }} / E_{\text {crit }}$. The possibility to reach such extreme intensity levels is of very much interest. However, it is clear that setting up the conditions necessary for vacuum light bullets to be formed, is a technological challenge.

Presently the electric fields that can be supported by the walls before field emission takes place are of the order $10^{8} \mathrm{~V} / \mathrm{m}[8]$. For such field strengths we note from the above estimates that we must have $k r_{\text {init }} \sim 10^{11}$, in which case either the initial beam radius becomes unrealistically large (of the order of $\mathrm{km}$ ), or the wavelength to short (i.e. in the short uv or x-ray regime) for the walls to be conducting. Two things should be stressed, however. Firstly it should be noted that the experimentally possible field level before field emission takes place have increased significantly in recent years [8]. Secondly, in our geometry there is no normal component of the electric field at the wall surfaces, which may allow for a higher (central) field strength than normal, relaxing the necessary value on $k r_{\text {init }}$. A slightly different but related technological question is whether the huge beam powers needed for vacuum self-focusing can be reached. This issue was discussed in Ref. [3], where it was concluded that it may occur within the next 10-15 years, given the current rate of technological improvement.

A very interesting question from a principal point of view, would be whether it is possible to have fully three-dimensional QED-structures which do not require any guiding support. However, it seems that this issue cannot be addressed within a perturbational approach, and further research is thus re- 
quired.

In this paper we have shown that the photon-photon scattering, due to the exchange of virtual electron-positron pairs, that effectively gives rise to selfinteraction terms in Maxwell's equations, can cause optical collapse in vacuum if the right conditions are met. More specifically, high intensity electromagnetic waves guided by two parallel conducting planes can form light bullets, which can collapse if the intensity of the beams are high enough. It is possible that higher order corrections to the Euler-Heisenberg Lagrangian can counteract such a collapse scenario, thus leading to stable optical vacuum structures. Further research is also necessary to find out more about how the optimum configurations can be changed, both in the present paper and in previous work (e.g. [3]).

\section{References}

[1] Heisenberg W. and Euler H., Z. Physik 98,714 (1936).

[2] Schwinger J., Phys. Rev. 82, 664 (1951).

[3] Soljacic M. and Segev M., Phys. Rev. A 62, 043817 (2000).

[4] Brodin G., Marklund M. and Stenflo L., Phys. Rev. Lett. 87171801 (2001).

[5] Desaix M., Anderson D. and Lisak M., J. Opt. Soc. Am. B 82082 (1991).

[6] Anderson D., Cattani F. and Lisak M., Phys. Scripta T82 32 (1999).

[7] Silberberg Y., Opt. Lett. 151282 (1990).

[8] Graber J., Ph.D. Dissertation (Cornell University, 1993), see also http://w4.lns.cornell.edu/public/CESR/SRF/BasicSRF/SRFBas1.html 